\title{
Initial coefficient bounds for certain class of meromorphic bi-univalent functions
}

\author{
Ahmad Zireh \\ Faculty of Mathematical Sciences, \\ Shahrood University of Technology, Iran \\ email: azireh@gmail.com
}

\author{
Safa Salehian \\ Faculty of Mathematical Sciences, \\ Shahrood University of Technology, Iran \\ email: gilan86@yahoo.com
}

\begin{abstract}
In this paper, we introduce and investigate an interesting subclass of meromorphic bi-univalent functions defined on $\Delta=\{z \in \mathbb{C}$ : $1<|z|<\infty\}$. For functions belonging to this class, estimates on the initial coefficients are obtained. The results presented in this paper generalize and improve some recent works.
\end{abstract}

\section{Introduction}

Let $\Sigma$ be the family of meromorphic functions $f$ of the form

$$
f(z)=z+b_{0}+\sum_{n=1}^{\infty} b_{n} \frac{1}{z^{n}},
$$

that are univalent in $\Delta=\{z \in \mathbb{C}: 1<|z|<\infty\}$. Since $\mathrm{f} \in \Sigma$ is univalent, it has an inverse $f^{-1}$ that satisfy

$$
f^{-1}(f(z))=z \quad(z \in \Delta)
$$

and

$$
f\left(f^{-1}(w)\right)=w \quad(M<|w|<\infty, M>0) .
$$

2010 Mathematics Subject Classification: 30C45, 30C50

Key words and phrases: meromorphic functions, meromorphic bi-univalent functions, coefficient estimates 
Furthermore, the inverse function $\mathrm{f}^{-1}$ has a series expansion of the form

$$
f^{-1}(w)=w+\sum_{n=0}^{\infty} B_{n} \frac{1}{w^{n}}
$$

where $M<|w|<\infty$. A simple calculation shows that the function $\mathrm{f}^{-1}$, is given by

$$
f^{-1}(w)=w-b_{0}-\frac{b_{1}}{w}-\frac{b_{2}+b_{0} b_{1}}{w^{2}}-\frac{b_{3}+2 b_{0} b_{1}+b_{0}^{2} b_{1}+b_{1}^{2}}{w^{3}}+\ldots .
$$

A function $f \in \Sigma$ is said to be meromorphic bi-univalent if $f^{-1} \in \Sigma$. The family of all meromorphic bi-univalent functions is denoted by $\Sigma_{\mathfrak{B}}$.

Estimates on the coefficient of meromorphic univalent functions were widely investigated in the literature; for example, Schiffer [8] obtained the estimate $\left|b_{2}\right| \leq 2 / 3$ for meromorphic univalent functions $f \in \Sigma$ with $b_{0}=0$ and Duren [2] proved that $\left|b_{n}\right| \leq 2 /(n+1)$ for $f \in \Sigma$ with $b_{k}=0,1 \leq k \leq n / 2$.

For the coefficients of inverses of meromorphic univalent functions, Springer [10] proved that

$$
\left|\mathrm{B}_{3}\right| \leq 1 \quad \text { and } \quad\left|\mathrm{B}_{3}+\frac{1}{2} \mathrm{~B}_{1}^{2}\right| \leq \frac{1}{2}
$$

and conjectured that

$$
\left|B_{2 n-1}\right| \leq \frac{(2 n-2) !}{n !(n-1) !} \quad(n=1,2, \cdots) .
$$

In 1977, Kubota [6] proved that the Springer conjecture is true for $n=3,4,5$ and subsequently Schober [9] obtained a sharp bounds for the coefficients $\mathrm{B}_{2 \mathrm{n}-1}, 1 \leq \mathrm{n} \leq 7$.

A function $f$ in the class $\Sigma_{\mathfrak{B}}$ is said to be memorphic bi-univalent starlike of order $\beta$ where $0 \leq \beta<1$, if it satisfies the flowing inequalities

$$
\operatorname{Re}\left(\frac{z f^{\prime}(z)}{f(z)}\right)>\beta \text { and } \operatorname{Re}\left(\frac{w g^{\prime}(w)}{g(w)}\right)>\beta(z, w \in \Delta),
$$

where $g$ is the inverse of $f$ given by $(3)$. We denote by $\Sigma_{\mathfrak{B}}^{*}(\beta)$ the class of all meromorphic bi-univalent starlike functions of order $\beta$. Similarly, a function $f$ in the class $\Sigma_{\mathfrak{B}}$ is said to be meromorphic bi-univalent strongly starlike of order $\alpha$ where $0<\alpha \leq 1$, if it satisfies the following conditions

$$
\left|\arg \left(\frac{z f^{\prime}(z)}{f(z)}\right)\right|<\frac{\alpha \pi}{2} \text { and }\left|\arg \left(\frac{w g^{\prime}(w)}{g(w)}\right) \arg \right|<\frac{\alpha \pi}{2} \quad(z, w \in \Delta),
$$


where $g$ is the inverse of $f$ given by (3). We denote by $\widetilde{\Sigma}_{\mathfrak{B}}^{*}(\alpha)$ the class of all meromorphic bi-univalent strongly starlike functions of order $\alpha$. The classes $\Sigma_{\mathfrak{B}}^{*}(\beta)$ and $\widetilde{\Sigma}_{\mathfrak{B}}^{*}(\alpha)$ were introduced and studied by Halim et al. [3].

Several researchers introduced and investigated some subclasses of meromorphically bi-univalent functions. (see, for detailes [3], [4], [5], [6], [9] and [13]).

Recently, Srivastava at al. [11] introduced the following subclasses of the meromorphic bi-univalent function and obtained non sharp estimates on the initial coefficients $\left|b_{0}\right|$ and $\left|b_{1}\right|$ as follow.

Definition 1 [11, Definition 2] A function $\mathrm{f}(\boldsymbol{z}) \in \Sigma_{\mathfrak{B}}$ given by (1) is said to be in the class $\Sigma_{\mathrm{B}, \lambda^{*}}(\alpha)$, if the following conditions are satisfied:

$$
\left|\arg \left(\frac{z\left[f^{\prime}(z)\right]^{\lambda}}{f(z)}\right)\right|<\frac{\alpha \pi}{2} \quad(0<\alpha \leq 1, \lambda \geq 1, z \in \Delta)
$$

and

$$
\left|\arg \left(\frac{w\left[g^{\prime}(w)\right]^{\lambda}}{g(w)}\right)\right|<\frac{\alpha \pi}{2} \quad(0<\alpha \leq 1, \lambda \geq 1, w \in \Delta),
$$

where the function $\mathrm{g}$ is the inverse of $\mathrm{f}$ given by (3).

Theorem 1 [11, Theorem 2.1] Let $\mathrm{f}(z) \in \Sigma_{\mathfrak{B}}$ given by (1) be in the class $\Sigma_{\mathrm{B}, \lambda^{*}}(\alpha)$. Then

$$
\left|b_{0}\right| \leq 2 \alpha, \quad\left|b_{1}\right| \leq \frac{2 \sqrt{5} \alpha^{2}}{1+\lambda}
$$

Definition 2 [11, Definition 3] A function $\mathrm{f}(z) \in \Sigma_{\mathfrak{B}}$ given by (1) is said to be in the class $\Sigma_{\mathrm{B}^{*}}(\lambda, \beta)$, if the following conditions are satisfied:

$$
\operatorname{Re}\left(\frac{z\left[f^{\prime}(z)\right]^{\lambda}}{f(z)}\right)>\beta \quad(0 \leq \beta<1, \lambda \geq 1, z \in \Delta)
$$

and

$$
\operatorname{Re}\left(\frac{w\left[g^{\prime}(w)\right]^{\lambda}}{g(w)}\right)>\beta \quad(0 \leq \beta<1, \lambda \geq 1, w \in \Delta)
$$

where the function $\mathrm{g}$ is the inverse of $\mathrm{f}$ given by (3). 
Theorem 2 [11, Theorem 3.1] Let $\mathbf{f}(\boldsymbol{z})$ given by $(1)$ be in the class $\Sigma_{\mathrm{B}^{*}}(\lambda, \beta)$. Then

$$
\left|b_{0}\right| \leq 2(1-\beta), \quad\left|b_{1}\right| \leq \frac{2(1-\beta) \sqrt{4 \beta^{2}-8 \beta+5}}{1+\lambda} .
$$

The following subclass of the meromorphic bi-univalent functions was investigated by Hai-Gen Xiao and Qing-Hua Xu [12].

Definition 3 [12, Definition 3] A function $\mathrm{f}(\boldsymbol{z}) \in \Sigma_{\mathfrak{B}}$ given by (1) is said to be in the class $\Sigma_{\vartheta}^{*}(\mu, \alpha)$, if the following conditions are satisfied:

$\left|\arg \left\{(1-\mu) \frac{z f^{\prime}(z)}{f(z)}+\mu\left(1+\frac{z f^{\prime \prime}(z)}{f^{\prime}(z)}\right)\right\}\right|<\frac{\alpha \pi}{2} \quad(0<\alpha \leq 1, \mu \in \mathbb{R}, z \in \Delta)$

and

$\left|\arg \left\{(1-\mu) \frac{w g^{\prime}(w)}{g(w)}+\mu\left(1+\frac{w g^{\prime \prime}(w)}{g^{\prime}(w)}\right)\right\}\right|<\frac{\alpha \pi}{2} \quad(0<\alpha \leq 1, \mu \in \mathbb{R}, w \in \Delta)$,

where the function $\mathrm{g}$ is the inverse of $\mathrm{f}$ given by (3).

Theorem 3 [12, Theorem 1] Let $\mathbf{f}(z)$ given by (1) be in the class $\Sigma_{\vartheta}^{*}(\mu, \alpha)$, $\mu \in \mathbb{R}-\left\{\frac{1}{2}, 1\right\}$. Then

$$
\left|b_{0}\right| \leq \frac{2 \alpha}{|1-\mu|}, \quad\left|b_{1}\right| \leq \frac{\sqrt{\mu^{2}-2 \mu+5}}{|1-\mu||2 \mu-1|} \alpha^{2} .
$$

The object of the present paper is to introduce a new subclass of the function class $\Sigma_{\mathfrak{B}}$ and obtain estimates on the initial coefficients for functions in this new subclass which improve Theorem 1, Theorem 2 and Theorem 3. Our results generalize and improve those in related works of several earlier authors.

\section{Coefficient bounds for the function class $M_{\Sigma_{\mathfrak{B}}}^{\mathrm{h}, \mathfrak{p}}(\lambda, \mu)$}

In this section, we introduce and investigate the general subclass $M_{\Sigma_{\mathfrak{B}}}^{\mathrm{h}, \mathfrak{p}}(\lambda, \mu)$.

Definition 4 Let the functions $\mathrm{h}, \mathrm{p}: \Delta \rightarrow \mathbb{C}$ be analytic functions and

$$
h(z)=1+\frac{h_{1}}{z}+\frac{h_{2}}{z^{2}}+\frac{h_{3}}{z^{3}}+\cdots, \quad p(z)=1+\frac{p_{1}}{z}+\frac{p_{2}}{z^{2}}+\frac{p_{3}}{z^{3}}+\cdots,
$$


such that

$$
\min \{\operatorname{Re}(h(z)), \operatorname{Re}(p(z))\}>0, \quad z \in \Delta .
$$

A function $\mathrm{f} \in \Sigma_{\mathfrak{B}}$ given by (1) is said to be in the class $M_{\Sigma_{\mathfrak{B}}}^{\mathrm{h}, \mathfrak{p}}(\lambda, \mu)(\lambda \geq 1$, $\mu \in \mathbb{R})$, if the following conditions are satisfied:

$$
(1-\mu) \frac{z\left(f^{\prime}(z)\right)^{\lambda}}{f(z)}+\mu\left(1+\frac{z f^{\prime \prime}(z)}{f^{\prime}(z)}\right)^{\lambda} \in h(\Delta) \quad(\lambda \geq 1, \mu \in \mathbb{R}, z \in \Delta)
$$

and

$$
(1-\mu) \frac{w\left(g^{\prime}(w)\right)^{\lambda}}{g(w)}+\mu\left(1+\frac{w g^{\prime \prime}(w)}{g^{\prime}(w)}\right)^{\lambda} \in p(\Delta) \quad(\lambda \geq 1, \mu \in \mathbb{R}, w \in \Delta),
$$

where the function $\mathrm{g}$ is the inverse of $\mathrm{f}$ given by (3).

Remark 1 There are many selections of the functions $\mathrm{h}(z)$ and $\mathrm{p}(\mathrm{z})$ which would provide interesting subclasses of the meromorphic function class $\Sigma$. For example, if we let

$$
h(z)=p(z)=\left(\frac{1+\frac{1}{z}}{1-\frac{1}{z}}\right)^{\alpha}=1+\frac{2 \alpha}{z}+\frac{2 \alpha^{2}}{z^{2}}+\cdots \quad(0<\alpha \leq 1, z \in \Delta),
$$

it is easy to verify that the functions $\mathrm{h}(\mathrm{z})$ and $\mathrm{p}(\mathrm{z})$ satisfy the hypotheses of Definition 4 .

If $\mathrm{f} \in \mathrm{M}_{\Sigma_{\mathfrak{B}}}^{\mathrm{h}, \mathrm{p}}(\lambda, \mu)$, then

$$
\begin{aligned}
&\left|\arg \left\{(1-\mu) \frac{z\left(f^{\prime}(z)\right)^{\lambda}}{f(z)}+\mu\left(1+\frac{z f^{\prime \prime}(z)}{f^{\prime}(z)}\right)^{\lambda}\right\}\right|<\frac{\alpha \pi}{2} \\
&(0<\alpha \leq 1, \lambda \geq 1, \mu \in \mathbb{R}, \quad z \in \Delta)
\end{aligned}
$$

and

$$
\begin{array}{r}
\left|\arg \left\{(1-\mu) \frac{w\left(g^{\prime}(w)\right)^{\lambda}}{g(w)}+\mu\left(1+\frac{w g^{\prime \prime}(w)}{g^{\prime}(w)}\right)^{\lambda}\right\}\right|<\frac{\alpha \pi}{2} \\
(0<\alpha \leq 1, \lambda \geq 1, \mu \in \mathbb{R}, w \in \Delta) .
\end{array}
$$

In this case, the function $\mathrm{f}$ is said to be in the class $M_{\Sigma_{\mathfrak{B}}}(\lambda, \mu, \alpha)$ and in special case $\lambda=1$, it reduces to Definition 3. We note that, by putting $\mu=0$, 
the class $\mathrm{M}_{\Sigma_{\mathfrak{B}}}(\lambda, \mu, \alpha)$ reduces to Definition 1 , the class $\Sigma_{\mathrm{B}, \lambda^{*}}(\alpha)$ introduced and studied by Srivastava et al. [11].

If we let

$$
\begin{aligned}
h(z) & =p(z)=\frac{1+\frac{1-2 \beta}{z}}{1-\frac{1}{z}} \\
& =1+\frac{2(1-\beta)}{z}+\frac{2(1-\beta)}{z^{2}}+\frac{2(1-\beta)}{z^{3}}+\ldots \quad(0 \leq \beta<1, z \in \Delta),
\end{aligned}
$$

it is easy to verify that the functions $\mathrm{h}(z)$ and $\mathrm{p}(z)$ satisfy the hypotheses of Definition 4 .

If $\mathrm{f} \in \mathrm{M}_{\Sigma_{\mathfrak{B}}}^{\mathrm{h}, \mathrm{p}}(\lambda, \mu)$, then

$$
\begin{aligned}
& \operatorname{Re}\left\{(1-\mu) \frac{z\left(f^{\prime}(z)\right)^{\lambda}}{f(z)}+\mu\left(1+\frac{z f^{\prime \prime}(z)}{f^{\prime}(z)}\right)^{\lambda}\right\}>\beta \\
& (0 \leq \beta<1, \lambda \geq 1, \mu \in \mathbb{R}, z \in \Delta)
\end{aligned}
$$

and

$$
\begin{aligned}
& \operatorname{Re}\left\{(1-\mu) \frac{w\left(g^{\prime}(w)\right)^{\lambda}}{g(w)}+\mu\left(1+\frac{w g^{\prime \prime}(w)}{g^{\prime}(w)}\right)^{\lambda}\right\}>\beta \\
&(0 \leq \beta<1, \lambda \geq 1, \mu \in \mathbb{R}, w \in \Delta) .
\end{aligned}
$$

Therefore for $\mathrm{h}(z)=\mathrm{p}(z)=\frac{1+\frac{1-2 \beta}{z}}{1-\frac{1}{z}}$ and $\mu=0$, the class $\mathrm{M}_{\Sigma_{\mathfrak{B}}}^{\mathrm{h}, \mathrm{p}}(\lambda, \mu)$ reduces to Definition 2.

Now, we derive the estimates of the coefficients $\left|b_{0}\right|$ and $\left|b_{1}\right|$ for class $M_{\Sigma_{\mathfrak{B}}}^{h, p}(\lambda, \mu)$.

Theorem 4 Let $\mathrm{f}(z) \in \Sigma_{\mathfrak{B}}$ given by (1) be in the class $M_{\Sigma_{\mathfrak{B}}}^{\mathrm{h}, \boldsymbol{p}}(\lambda, \mu)(\lambda \geq 1, \mu \in$ $\mathbb{R}-\{1\},(3 \lambda \mu+\mu-\lambda) \neq 1)$. Then

$$
\left|b_{0}\right| \leq \min \left\{\sqrt{\frac{\left|h_{1}\right|^{2}+\left|p_{1}\right|^{2}}{2(1-\mu)^{2}}}, \sqrt{\frac{\left|h_{2}\right|+\left|p_{2}\right|}{2|1-\mu|}}\right\}
$$

and

$$
\left|b_{1}\right| \leq \min \left\{\frac{\left|h_{2}\right|+\left|p_{2}\right|}{2|3 \lambda \mu+\mu-\lambda-1|}, \frac{1}{|3 \lambda \mu+\mu-\lambda-1|} \sqrt{\frac{\left|h_{2}\right|^{2}+\left|p_{2}\right|^{2}}{2}+\frac{\left(\left|h_{1}\right|^{2}+\left|p_{1}\right|^{2}\right)^{2}}{4(1-\mu)^{2}}}\right\} .
$$


Proof. First of all, we write the argument inequalities in (4) and (5) in their equivalent forms as follows:

$$
(1-\mu) \frac{z\left(f^{\prime}(z)\right)^{\lambda}}{f(z)}+\mu\left(1+\frac{z f^{\prime \prime}(z)}{f^{\prime}(z)}\right)^{\lambda}=h(z) \quad(z \in \Delta)
$$

and

$$
(1-\mu) \frac{w\left(g^{\prime}(w)\right)^{\lambda}}{g(w)}+\mu\left(1+\frac{w g^{\prime \prime}(w)}{g^{\prime}(w)}\right)^{\lambda}=p(w) \quad(w \in \Delta),
$$

respectively, where functions $h(z)$ and $p(w)$ satisfy the conditions of Definition 4.

Furtheremore, the functions $h(z)$ and $p(w)$ have the forms:

$$
h(z)=1+\frac{h_{1}}{z}+\frac{h_{2}}{z^{2}}+\frac{h_{3}}{z^{3}}+\cdots
$$

and

$$
p(w)=1+\frac{p_{1}}{w}+\frac{p_{2}}{w^{2}}+\frac{p_{3}}{w^{2}}+\cdots,
$$

respectively. Now, upon equating the coefficients of

$$
\begin{gathered}
(1-\mu) \frac{z\left(f^{\prime}(z)\right)^{\lambda}}{f(z)}+\mu\left(1+\frac{z f^{\prime \prime}(z)}{f^{\prime}(z)}\right)^{\lambda} \\
=1-\frac{(1-\mu) b_{0}}{z}+\frac{(1-\mu) b_{0}^{2}+(3 \lambda \mu+\mu-\lambda-1) b_{1}}{z^{2}}+\ldots
\end{gathered}
$$

with those of $h(z)$ and coefficients of

$$
\begin{gathered}
(1-\mu) \frac{w\left(g^{\prime}(w)\right)^{\lambda}}{g(w)}+\mu\left(1+\frac{w g^{\prime \prime}(w)}{g^{\prime}(w)}\right)^{\lambda} \\
=1+\frac{(1-\mu) b_{0}}{w}+\frac{(1-\mu) b_{0}^{2}-(3 \lambda \mu+\mu-\lambda-1) b_{1}}{w^{2}}+\ldots
\end{gathered}
$$

with those of $p(w)$, we get

$$
\begin{aligned}
-(1-\mu) b_{0} & =h_{1}, \\
(1-\mu) b_{0}^{2}+(3 \lambda \mu+\mu-\lambda-1) b_{1} & =h_{2}, \\
(1-\mu) b_{0} & =p_{1}
\end{aligned}
$$


and

$$
(1-\mu) b_{0}^{2}-(3 \lambda \mu+\mu-\lambda-1) b_{1}=p_{2}
$$

From (12) and (14), we get

$$
h_{1}=-p_{1} \quad\left(b_{0}=-\frac{h_{1}}{1-\mu}\right)
$$

and

$$
2(1-\mu)^{2} b_{0}^{2}=h_{1}^{2}+p_{1}^{2} .
$$

Adding (13) and (15), we get

$$
2(1-\mu) b_{0}^{2}=h_{2}+p_{2} .
$$

Therefore, we find from the equations (16) and (17) that

$$
\left|b_{0}\right|^{2} \leq \frac{\left|h_{1}\right|^{2}+\left|p_{1}\right|^{2}}{2(1-\mu)^{2}}
$$

and

$$
\left|b_{0}\right|^{2} \leq \frac{\left|h_{2}\right|+\left|p_{2}\right|}{2|1-\mu|}
$$

respectively. So we get the desired estimate on the coefficient $\left|b_{0}\right|$ as asserted in (6).

Next, in order to find the bound on the coefficient $\left|b_{1}\right|$, we subtract (15) from (13). We thus get

$$
2(3 \lambda \mu+\mu-\lambda-1) b_{1}=h_{2}-p_{2} .
$$

By squaring and adding (13) and (15), using (16) in the computation leads to

$$
b_{1}^{2}=\frac{1}{2(3 \lambda \mu+\mu-\lambda-1)^{2}}\left(h_{2}^{2}+p_{2}^{2}-\frac{\left(h_{1}^{2}+p_{1}^{2}\right)^{2}}{2(1-\mu)^{2}}\right) .
$$

Therefore, we find from the equations (18) and (19) that

$$
\left|b_{1}\right| \leq \frac{\left|h_{2}\right|+\left|p_{2}\right|}{2|3 \lambda \mu+\mu-\lambda-1|}
$$

and

$$
\left|b_{1}\right| \leq \frac{1}{|3 \lambda \mu+\mu-\lambda-1|} \sqrt{\frac{\left|h_{2}\right|^{2}+\left|p_{2}\right|^{2}}{2}+\frac{\left(\left|h_{1}\right|^{2}+\left|p_{1}\right|^{2}\right)^{2}}{4(1-\mu)^{2}}} .
$$

This evidently completes the proof of Theorem 4 . 


\section{Corollaries and consequences}

By setting

$h(z)=p(z)=\frac{1+\frac{1-2 \beta}{z}}{1-\frac{1}{z}}=1+\frac{2(1-\beta)}{z}+\frac{2(1-\beta)}{z^{2}}+\ldots \quad(0 \leq \beta<1, z \in \Delta)$ and $\mu=0$ in Theorem 4 , we conclude the following result.

Corollary 1 Let the function $\mathrm{f}(z)$ given by $(1)$ be in the class $\Sigma_{\mathrm{B}^{*}}(\lambda, \beta),(0 \leq$ $\beta<1, \lambda \geq 1$ ). Then

$$
\left|b_{0}\right| \leq \begin{cases}\sqrt{2(1-\beta)} ; & \beta \leq \frac{1}{2} \\ 2(1-\beta) ; & \beta>\frac{1}{2}\end{cases}
$$

and

$$
\left|b_{1}\right| \leq \min \left\{\frac{2(1-\beta)}{1+\lambda}, \frac{2(1-\beta) \sqrt{4 \beta^{2}-8 \beta+5}}{1+\lambda}\right\}=\frac{2(1-\beta)}{1+\lambda} .
$$

Remark 2 The bounds on $\left|\mathrm{b}_{0}\right|$ and $\left|\mathrm{b}_{1}\right|$ given in Corollary 1 are better than those given in Theorem 2.

By setting $\lambda=1$ in Corollary 1 , we conclude the following result.

Corollary 2 Let the function $\mathrm{f}(\boldsymbol{z})$ given by $(1)$ be in the class $\Sigma_{\mathfrak{B}}^{*}(\beta)(0 \leq$ $\beta<1)$. Then

$$
\left|b_{0}\right| \leq \begin{cases}\sqrt{2(1-\beta)} ; & \beta \leq \frac{1}{2} \\ 2(1-\beta) ; & \beta>\frac{1}{2}\end{cases}
$$

and

$$
\left|b_{1}\right| \leq \min \left\{1-\beta,(1-\beta) \sqrt{1+4(1-\beta)^{2}}\right\}=1-\beta .
$$

Remark 3 The bounds on $\left|\mathrm{b}_{0}\right|$ and $\left|\mathrm{b}_{1}\right|$ given in Corollary 2 are better than those given by Halim et al. [3, Theorem 1].

By setting

$$
h(z)=p(z)=\left(\frac{1+\frac{1}{z}}{1-\frac{1}{z}}\right)^{\alpha} \quad(0<\alpha \leq 1, \quad z \in \Delta),
$$

in Theorem 4, we conclude the following result. 
Corollary 3 Let the function $f(z)$ given by (1) be in the class $M_{\Sigma_{\mathfrak{B}}}(\lambda, \mu, \alpha)$ $(0<\alpha \leq 1, \lambda \geq 1, \mu \in \mathbb{R}-\{1\},(3 \lambda \mu+\mu-\lambda) \neq 1)$. Then

$$
\left|b_{0}\right| \leq \begin{cases}\alpha \sqrt{\frac{2}{\mid 1-\mu} ;} ; & |1-\mu| \leq 2 \\ \frac{2 \alpha}{|1-\mu|} ; & |1-\mu|>2\end{cases}
$$

and

$$
\begin{aligned}
\left|b_{1}\right| & \leq \min \left\{\frac{2 \alpha^{2}}{|3 \lambda \mu+\mu-\lambda-1|}, \frac{2 \alpha^{2}}{|3 \lambda \mu+\mu-\lambda-1|} \sqrt{1+\frac{4}{(1-\mu)^{2}}}\right\} \\
& =\frac{2 \alpha^{2}}{|3 \lambda \mu+\mu-\lambda-1|} .
\end{aligned}
$$

By setting $\mu=0$ in Corollary 3 , we conclude the following result.

Corollary 4 Let the function $\mathrm{f}(\boldsymbol{z})$ given by $(1)$ be in the class $\Sigma_{\mathrm{B}, \lambda^{*}}(\alpha)(0<$ $\alpha \leq 1, \lambda \geq 1)$. Then

$$
\left|\mathrm{b}_{0}\right| \leq \sqrt{2} \alpha
$$

and

$$
\left|b_{1}\right| \leq \frac{2 \alpha^{2}}{\lambda+1}
$$

Remark 4 The bounds on $\left|\mathrm{b}_{0}\right|$ and $\left|\mathrm{b}_{1}\right|$ given in Corollary 4 are better than those given in Theorem 2.

By setting $\lambda=1$ in Corollary 3, we conclude the following result.

Corollary 5 Let the function $\mathrm{f}(z)$ given by (1) be in the class $\Sigma_{\vartheta}^{*}(\mu, \alpha)(0<$ $\left.\alpha \leq 1, \mu \in \mathbb{R}-\left\{\frac{1}{2}, 1\right\}\right)$. Then

$$
\left|b_{0}\right| \leq \begin{cases}\alpha \sqrt{\frac{2}{\mid 1-\mu} ;} ; & |1-\mu| \leq 2 \\ \frac{2 \alpha}{|1-\mu|} ; & |1-\mu|>2\end{cases}
$$

and

$$
\left|b_{1}\right| \leq \min \left\{\frac{\alpha^{2}}{|2 \mu-1|}, \frac{\sqrt{\mu^{2}-2 \mu+5}}{|1-\mu||2 \mu-1|} \alpha^{2}\right\}=\frac{\alpha^{2}}{|2 \mu-1|}
$$


Remark 5 The bounds on $\left|\mathrm{b}_{0}\right|$ and $\left|\mathrm{b}_{1}\right|$ given in Corollary 5 are better than those given in Theorem 3.

By setting $\mu=0$ in Corollary 5 , we conclude the following result.

Corollary 6 Let the function $\mathrm{f}(\boldsymbol{z})$ given by (1) be in the class $\widetilde{\Sigma}_{\mathfrak{B}}^{*}(\alpha)(0<$ $\alpha \leq 1)$. Then

$$
\left|b_{0}\right| \leq \sqrt{2} \alpha \quad \text { and } \quad\left|b_{1}\right| \leq \min \left\{\alpha^{2}, \sqrt{5} \alpha^{2}\right\}=\alpha^{2}
$$

Remark 6 The bounds on $\left|\mathrm{b}_{0}\right|$ and $\left|\mathrm{b}_{1}\right|$ given in Corollary 6 are better than those given by Halim et al. [3, Theorem 2].

\section{Acknowledgments}

The authors wish to thank the referee, for the careful reading of the paper and for the helpful suggestions and comments.

\section{References}

[1] E. Deniz, Certain subclasses of bi-univalent functions satisfying subordinate conditions, J. Class. Anal., 2 (1) (2013), 49-60.

[2] P. L. Duren, Univalent functions, Grundlehren der Mathematischen Wissenschaften, Band 259, Springer-Verlag, New York, Berlin, Heidelberg and Tokyo, 1983.

[3] S. A. Halim, S. G. Hamidi, V. Ravichandran, Coefficient estimates for meromorphic bi-univalent functions, arXiv:1108.4089v1 (2011), 1-9.

[4] S. G. Hamidi, S. A. Halim, J. M. Jahangiri, Coefficient estimates for a class of meromorphic biunivalent functions, C. R. Acad. Sci. Paris Sr., 351 (2013), 349-352.

[5] G. P. Kapoor, A. K. Mishra, Coefficient estimates for inverses of starlike functions of positive order, J. Math. Anal. Appl., 329 (2) (2007), 922-934.

[6] Y. Kubota, Coefficients of meromorphic univalent functions, Kodai Math. Sem. Rep., 28 (1976/77), 253-261. 
[7] S. Salehian and A. Zireh, Coefficient estimates for certain subclass of meromorphic and bi-univalent functions, Commun. Korean Math. Soc., 32 (2) (2017), 389-397.

[8] M. Schiffer, Sur un problème d'extrémum de la représentation conforme, Bull. Soc. Math. France, 66 (1938), 48-55.

[9] G. Schober, Coefficients of inverses of meromorphic univalent functions, Proc. Amer. Math. Soc., 67 (1) (1977), 111-116.

[10] G. Springer, The coefficient problem for schlicht mappings of the exterior of the unit circle, Trans. Amer. Math. Soc., 70 (1951), 421-450.

[11] H. M. Srivastava, S. B. Joshi, S. S. Joshi, H. Pawar, Coefficient estimates for certain subclasses of memorphically bi-univalent function, Palest. J. Math, 5(1) (2016), 250-258.

[12] H.-G. Xiao, Q.-H. Xu, Coefficient estimates for three generalized classes of meromorphic and bi-univalent functions, Filomat, 29 (7) (2015), 16011612 .

[13] Q.-H. Xu, C-B Lv, H.M. Srivastava, Coefficient estimates for the inverses of a certain general class of spirallike functions, Appl. Math. Comput., 219 (2013), 7000-7011.

[14] A. Zireh, E. Analouei Adegani, S. Bulut, Faber polynomial coefficient estimates for a comprehensive subclass of analytic bi-univalent functions defined by subordination, Bull. Belg. Math. Soc. Simon Stevin., 23 (2016), 487-504.

[15] A. Zireh, E. Analouei Adegani, M. Bidkham, Faber polynomial coefficient estimates for subclass of bi-univalent functions defined by quasisubordinate, Math. Slovaca, 68 (2018), 369-378. 